\title{
S V M Based Hybrid Classification and Information Extraction from Hyperspectral Images for Precision Agriculture
}

\author{
Anil YR ${ }^{1}$, Dr. Rehna VJ ${ }^{2}$ \\ ${ }^{1}$ M.Tech, VLSI Design and Embedded Systems \\ ${ }^{2}$ Professor and HOD, VLSI Design and Embedded Systems, M S Engineering College, India. \\ anilyrreddy@gmail.com; rehnavj09@gmail.com
}

\begin{abstract}
In the recent decades there is a lot of fear in natural resources than of man-made resources. Analysis and observation of Agricultural area, human living area/urban area and water bodies has become crucial. We need to know how much amount of land is used and how much amount of land is not used in global scale. There is a constant change in the geography of earth due to land erosion, biological and geo chemical cycling and bio-diversity. As there is a constant change we need to know the land used and land not used. Knowing these shows a prominent role in global \& social development of earth. There is a rapid change on the surface of the earth which has to be noticed and recorded. All these changes on the surface of the earth can be noticed by remote sensing. Remote sensing has become a vital means for observing changes, particularly as a means of completing or updating conventional data gathering techniques. [1] In the past few years people have gained knowledge about hyperspectral images and their importance in the field of remote sensing area. The basic meaning of "hyper-spectral" means "hyper" = "over" that means "too many" which depicts the huge number of measured wave length bands and "spectral-spectra" means relating to spectrum. This paper mainly describes the computational way to execute S V M and the other main highlight of the paper is to detect edges of classified images and real time area estimation of agriculture area, human living area and water bodies for all Hyperspectral images.
\end{abstract}

Keywords: Hyperspectral Images, Support Vector Machine, Hybrid Classification, Agriculture Area, Human Living Area, Water bodies, Edge Detection, Area Estimation.

\section{INTRODUCTION}

The latest apprehension of sustainable use of natural assets, monitoring and analysis of agriculture area, human living area and water bodies Analysis and observation of Agricultural area, human living area/urban area and water bodies has become crucial. We need to know how much amount of land is used and how much amount of land is not used in global scale. There is a constant change in the geography of earth due to land erosion, biological and geo chemical cycling and bio-diversity. As there is a constant change we need to know the land used and land not used. Knowing these shows a prominent role in global \& social development of earth. There is a rapid change on the surface of the earth which has to be noticed and recorded. All these changes on the surface of the earth can be noticed by remote sensing. Remote sensing has become a vital means for observing changes, particularly as a means of completing or updating conventional data gathering techniques.

\section{SUPPORT VECTOR MACHINE}

Basically we take a hyper-spectral image then we will remove noise which is also called as glitch in hyper-spectral images. Once the noise is removed using a low pass filter we then proceed with classification of images. 
Image classification is done by S V M. In the current research Support Vector Machine was matched with other classification approaches like, maximum likelihood and artificial neural networks classifiers for remote sensing imagery and it has outdid all of the in toughness and precision. So we can say S V M is one of the best classifier in the recent trends. [2][3]

Support Vector Machine has a greater prospective, mainly for hyper-spectral samples, due to their great dimensionality. S V M based classification of hyper-spectral image into agriculture area, human living area and water bodies.

\section{IMPLEMENTATION AND METHODLOGY}

Hyper-spectral images act as the input images. Once we get the hyper-spectral images, we need to preprocess those images to remove glitches and then classification is done based on the algorithm classed support vector machine.

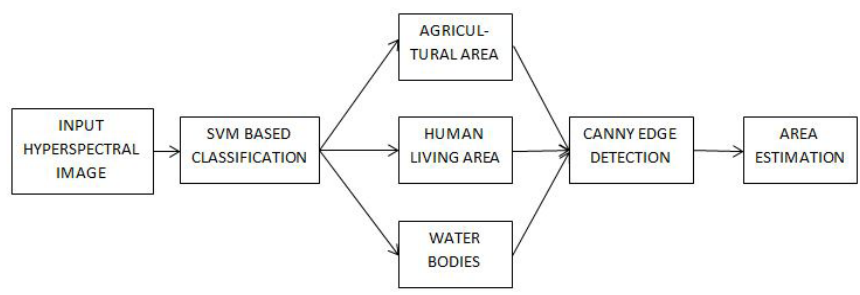

Above figure shows the generalized block diagram of S V M based classification for hyper-spectral images.

The hyper plane will be created in S V M to distinguish between the objects; these hyper planes will be created with the help of support vectors, and these support vectors will be created when there are different shades, texture, reflection and absorption in the given hyper-spectral image. The support vectors have to be educated and theses educated / trained vectors classify the images, vectors quantities are those with have magnitude and direction. The S V M method pursues to locate ultimate setting apart hyper plane among instructions using aiming on education instances which might be positioned at the brink of the elegance elements. This education types are known as help "vectors". The training cases other than support vectors will be rejected. This is how the process continues, now not most effective is a top rated hyper plane equipped, out additionally few training cases are effectively used; therefore high category precision is finished with small training sets. Pixel based schemes are used to find the support vectors and this is how we train the support vectors and using these support vectors we classify the images.

Now let us understand how the support vectors are calculated based on the images and how a hyper plane is used to decide the difference in the objects of the hyper-spectral images. The execution of the primarily based image estimation is done, allowing us to understand how supervised binary classification is solved. Consider the trained cases are symbolized by $[x i, y i] i=1,2, \ldots, N, \&$ yi $\in[-1,+1]$, in which $N$ is the most important parameter that represents the number of trained cases, yi $=+1$ for class $\omega 1$ and $y i=-1$ for class $\omega 2$. Assume the two classes are linearly separable. The vector quantities $\mathrm{w}$ and $\mathrm{w} 0$, are used to decide the hyper plane which help in separating the different objects in the images. Now let's take these vectors quantities with a function $\mathrm{F}(\mathrm{x})$, which is given in the equation 1 below.

$\mathrm{F}(\mathrm{x})=\mathrm{w} * \mathrm{x}+\mathrm{w} 0=0$

Now we need to find the hyper plane, theses plane can found by $w 0$ and $w 1$, it is represented as yi $(w * x i+w 0) \geq$ +1 for $y i=+1$ it's for class $\omega 1$ and yi $\left(w^{*} x i+w 0\right) \leq-1$ for $y i=-1$ and this is for class $\omega 2$. The above equation can be joined together to form the equation 2 . 
S V M Based Hybrid Classification and Information Extraction from Hyperspectral Images for Precision Agriculture

yi $\left(w^{*} x i+w 0\right)-1 \geq 0$

There may be many hyper planes that are separated based the training give to them, hyper planes may be many, like superior hyper plane can do the better classification. The main aim is to look for the hyper plane which leaves the extreme margin among the peers in an effort to get the uppermost worth hyperplane. The support vectors lie between two hyper planes which will be adjacent to the finest and it is given by the equation 3 .

$\mathrm{W} * \mathrm{xi}+\mathrm{w} 0= \pm 1$

If a simple/test cases are rescaled according to the hyper plane coefficients $\mathrm{w}$ and $\mathrm{w} 0$ takes place, the margin can be expressed as $2 /\|\mathrm{W}\|$. The ideal hyper plane can be originated by explaining the next optimization equation 4.

Reducing/minimizing $1 / 2\left\|w^{2}\right\|$

The above equation is focus on yi $(w * x i+w 0)-1 \geq 0 i=0,1, \ldots N$

Using a Lagrangian formula, the above problematic case can be solved as:

$$
\text { Reducing } \sum_{i=1}^{N} \lambda \mathbf{i}-\frac{1}{2} \sum_{i, j=1}^{N} \lambda_{i} \lambda_{j} \text { yi yj }(x i * x j)
$$

which is subjected to $\quad \sum_{\mathrm{i}=1}^{\mathrm{N}}(\lambda \mathrm{i}$ yi $)=0 \& \lambda \mathrm{i}>0, \mathrm{i}=1,2 \ldots . \mathrm{N}$

$\lambda i$ (lambda) is the Lagrange multipliers. There many such multiples given by Mr. Lagrange

Under these formulas, the optimal hyper plane discriminant of the function will become:

$$
\mathrm{f}(\mathrm{x})=\sum_{\mathrm{i} \mathrm{e}_{\mathrm{s}}}^{\infty} \lambda \mathrm{i} \mathrm{yi}(\mathrm{xi} \mathrm{x})+\mathrm{w} 0
$$

Wherein $\mathrm{S}$ will be the subset of trained cases that relate to non zero Lagrange approach. The assist vectors are the trained sample which we will be using for our classification. In many cases the samples will not be linearly distinguishable as a result the equation 2 cannot be used. Now we need to take in consideration the intensification of margin and minimization of errors case, slack variable i.e. $\xi$ will be used in the equation 7 and the features are defined as:

Reducing $\mathbf{J}(\mathbf{w}, \mathbf{w O}, \xi)=1 / 2\left\|\mathbf{w}^{2}\right\|+C \sum_{i=1}^{\mathrm{N}}(\xi \mathbf{i})$

The above equation will be focus to $y i\left(w^{*} \mathrm{x}+\mathrm{w} 0\right) \geq 1-\xi \mathrm{i}$

Now we need simplify the above equation to non linear competencies, to do the we need the help of the vector quantities like $\mathrm{x}$ and which is a multi dimensional in space. Now the finest hyper plane will be created to separate the objects in the hyper-spectral images. The Mercer's statement says that when there are high dimensional utility area might add extra intricacy to the problem. So to resolve the problem we consider the internal product of action has an equal depiction given in the equation 8.

$\Phi(\mathrm{x}) \Phi(\mathrm{z})=\mathrm{K}(\mathrm{x}, \mathrm{z})$

The Kernel quantity is given by $\mathrm{K}(\mathrm{x}, \mathrm{z})$ and it is called as kernel function. Now we need to find the kernel function $\mathrm{K}$, the value of $\mathrm{K}$ can be found by training the obvious method of $\Phi$.

The double optimization difficulty can be formed as shown in then equation 9:

$$
\text { Reducing } \sum_{\mathrm{i}=1}^{\mathrm{N}} \lambda \mathrm{i}-\frac{1}{2} \sum_{\mathrm{i}, \mathrm{j}=1}^{\mathrm{N}} \lambda \mathrm{i} \lambda \mathrm{j} \text { yi yj } \mathrm{K}(\mathrm{xi} * \mathrm{xj})
$$

which is subjected to $\sum_{\mathrm{i}=1}^{\mathrm{N}}\left(\lambda_{\mathrm{i}} \mathrm{yi}\right)=0 \quad \& \quad \lambda_{\mathrm{i}}>0, \mathrm{i}=1,2 \ldots . \mathrm{N}$

The subsequent equation becomes as follows:

$$
f(x)=\sum_{i \in s}^{\infty} \lambda i y i K(x i x)+w 0
$$


S V M Based Hybrid Classification and Information Extraction from Hyperspectral Images for Precision Agriculture

Average,the S V M type approach become determined very capable for object-based totally photograph evaluation. In many cases it is proven that it could yield equivalent or maybe higher effects than the adjacent neighbor for supervised class.

The performance of S V M changed into notable, with only a few minutes of runtime necessary for scheduling. These turned into hypothetically projected however additionally, the execution in $\mathrm{C}++$ is extraordinarily rapid. But very big faraway sensing data sets had been now not to be tested. A superb characteristic of S V M is a small education set is wanted to offer best results, due to the fact simplest the guide vectors are of importance throughout training.

\section{Canny Edge Detection and Area Estimation}

The motive of part recognition in trendy is to noticeably decrease the quantity of records in an photo, whilst protective the structural houses to be used for in addition photographs processing. Numerous theories are already present, and this work sheet specializes in a selected one evolved by Mr. John F Canny in 1986. Even though it is quite old, it has end up one in all the standard area detection techniques and its miles nonetheless utilized in studies [5]. The Canny part detection approaches have five steps to be followed:

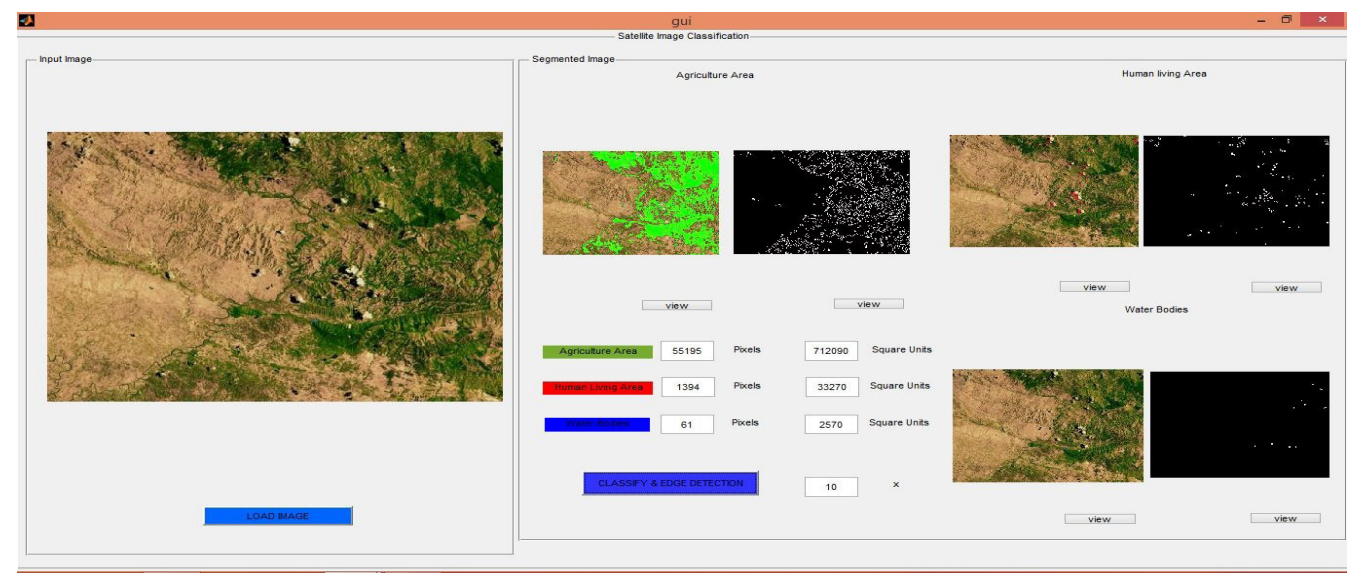

Fig1. Hyperspectral image is been classified into agriculture area, human living area and water bodies and edge detection for the same hasbeen done.

- Smoothing: Concealing of the image to dispose of the noise.

- Locating gradients: the rims need to be marked wherein the inclines of the picture has huge extents.

- Non maximum suppression: handiest local maxima ought to be noticeable as edges.

- Doubling threshold: capacity edges are decided by using thresholding.

- Monitoring through hysteresis: very last edges are decided by way of destroying all edges that aren't related to a very sure area.

\section{RESUlts AND ConCLUSiON}

A real time hyperspectral image is taken and it's been classified into agriculture area, human living area and water bodies based on support vector machine and once the classification is done the classified images are processed on Canny's edge detection to get the edges for the respective areas, later the area is calculated based on the pixels and the edges. The table shows the area in square units multiplied by the variable factor X. The factor $\mathrm{X}$ is the distance between the camera and the surface of the earth. 
S V M Based Hybrid Classification and Information Extraction from Hyperspectral Images for Precision Agriculture

\begin{tabular}{|c|c|c|}
\hline $\begin{array}{c}\text { Classified and Edge detected } \\
\text { Hyperspectral image }\end{array}$ & Number of Pixels & $\begin{array}{c}\text { Area in square units } \\
\text { multiplied with the factor X }\end{array}$ \\
\hline Agriculture area (green) & 55195 & 712090 \\
\hline Human living area (red) & 1394 & 33270 \\
\hline Area of Water bodies (blue) & 61 & 2570 \\
\hline
\end{tabular}

\section{ACKNOWLEDGMENT}

The authors would like to acknowledge Principal and management of MS Engineering College Bengaluru for their assistance and support.

\section{REFERENCES}

1. Clement Chion, Jacques Landry and Costa 2008 "A genetic programming based method for hyperspectral data information extraction: agriculture applications". IEEE transaction on geoscience and remote sensing vol 46no 8 august 2008

2. S. Natya and VJ Rehna "Land cover classification schemes using remort sensing images: A recent survey" British Journal of applied science and technology 13940:1-11,2016,doi: 10.9734/bjast/2016/22037

3. Pavan Kumar, binay Kumar Singh, and Meenu Rani 2013 An Efficient Hybrid Classification Approach for Land Use/Land Cover Analysis in a Semi-Desert Area Using ETM+ and LISS-III Sensor" IEEE SENSORS JOURNAL, VOL. 13, NO. 6, JUNE 20132161

4. Jianhong Liu, Wenquan Zhu*, Minjie Mou, Lingli Wang "Cropland Parcels Extraction based on Texture Analysis and Multi-spectral Image Classification" IEEE SENSORS JOURNAL, VOL. 16, NO. 7, JUNE 2014 2161

5. John Canny. A Computational approcah to edge detection. Pattran analysis and Machine Intelligence, IEEE Transaction on, PAMI-8(6):679-698, Nov.1986

6. Farid Melgani, member IEEE, and Lorenzo bruzzone, senior member IEEE "Classification of hyperspectral remort sensing images with support vector machines". IEEE transaction on geoscience and remote sensing vol 42 no 8 august 2004

7. A support vector machine approach for object based image analysis by Angelos Tzotsos, Departmet of surveying, National Technical university of Athens Greece

8. A Carlson, Z Guo, S Balasubramanian, L.-T. Pang, T.J. King Liu and B. Nikolic, "FinFET SRAM with Enhanced Read / Write Margins", IEEE International SOI Conference Proceedings, ISBN 1-4244-0289-1, 2006.

9. Young Bok Kim, Yong-Bin Kim, Lombardi, Fabrizio and Young Jun Lee, "A Low Power 8T SRAM Cell Design Technique for CNTFET", International SOC Design Conference 978-1-4244-2598-3, 24-25 November 2008.

Citation: Anil YR, Dr. Rehna VJ. "S V M Based Hybrid Classification and Information Extraction from Hyperspectral Images for Precision Agriculture", American Research Journal of Electronics and Communications Engineering; Vol 1, no 1; pp: 11-15.

Copyright (C) Anil YR, Dr. Rehna VJ. This is an open access article distributed under the Creative Commons Attribution License, which permits unrestricted use, distribution, and reproduction in any medium, provided the original work is properly cited. 